\title{
Disjoint and simultaneous hypercyclic Rolewicz-type operators
}

\author{
Nurhan Çolakoğlu ${ }^{1}$, Özgür Martin*2 (D) \\ ${ }^{1}$ Department of Mathematics, Istanbul Technical University, Maslak, 34469, Istanbul, Turkey \\ ${ }^{2}$ Department of Mathematics, Mimar Sinan Fine Arts University, Silahşör Cad. 71, Bomonti Şişli 34380, \\ Istanbul, Turkey
}

\begin{abstract}
We characterize disjoint hypercyclic and supercyclic tuples of unilateral Rolewicz-type operators on $c_{0}(\mathbb{N})$ and $\ell^{p}(\mathbb{N}), p \in[1, \infty)$, which are a generalization of the unilateral backward shift operator. We show that disjoint hypercyclicity and disjoint supercyclicity are equivalent among a subfamily of these operators and disjoint hypercyclic unilateral Rolewicz-type operators always satisfy the Disjoint Hypercyclicity Criterion. We also characterize simultaneous hypercyclic unilateral Rolewicz-type operators on $c_{0}(\mathbb{N})$ and $\ell^{p}(\mathbb{N}), p \in[1, \infty)$.
\end{abstract}

Mathematics Subject Classification (2020). 47A16, 47B37, 46B45

Keywords. hypercyclic vectors, hypercyclic operators, disjoint hypercyclicity, simultaneous hypercyclicity

\section{Introduction}

Let $\mathbb{N}$ denote the set of positive integers, $X$ be a separable and infinite dimensional Banach space over the real or complex scalar field $\mathbb{K}$, and let $\mathcal{B}(X)$ denote the algebra of bounded linear operators on $X$. An operator $T \in \mathcal{B}(X)$ is called hypercyclic if there exists $x \in X$ such that $\left\{T^{n} x: n \in \mathbb{N}\right\}$ is dense in $X$ and such a vector $x$ is said to be a hypercyclic vector for $T$.

The first example of a hypercyclic operator on a Banach space was given in 1969 by Rolewicz [11], who showed that if $B$ is the unweighted unilateral backward shift on $\ell^{2}(\mathbb{N})$, then $\lambda B$ is hypercyclic if and only if $|\lambda|>1$. Recall that $B$ is defined as $B e_{n}=e_{n-1}$, for $n \geq 2$ and $B e_{1}=0$ where $\left\{e_{j}: j \geq 1\right\}$ is the canonical basis.

One can generalize these operators to unilateral weighted shifts by multiplying the shifted vector by a weight sequence $\left(w_{n}\right)_{n \in \mathbb{N}}$ of scalars in $\mathbb{K}$, that is, $B_{w} e_{n}=w_{n} e_{n-1}$, for $n \geq 2$ and $B_{w} e_{1}=0$. In a fundamental paper in the area, Salas [12] completely characterized the hypercyclic unilateral weighted backward shifts on $\ell^{p}$ with $1 \leq p<\infty$ in terms of their weight sequences.

Another way to generalize Rolewicz's operators is to change the way these operators shift the vectors. In 2015, Bongiorno, Darji, and Di Piazza [6] studied the dynamics of

\footnotetext{
*Corresponding Author.

Email addresses: colakn@itu.edu.tr (N. Çolakoğlu), ozgur.martin@msgsu.edu.tr (Ö. Martin)

Received: 07.09.2020; Accepted: 13.06.2021
} 
operators which they call as the Rolewicz-type operators: Let the Rolewicz-type operator $\lambda T_{f}: X \rightarrow X$ be defined as

$$
\lambda T_{f} x=\lambda T_{f}\left(x_{1}, x_{2}, \ldots\right)=\left(\lambda x_{f(1)}, \lambda x_{f(2)}, \ldots\right),
$$

where $\lambda \in \mathbb{K}, X=c_{0}$ or $\ell^{p}(\mathbb{N}), 1 \leq p<\infty$, and $f: \mathbb{N} \rightarrow \mathbb{N}$ is a strictly increasing function with $f(1) \neq 1$. It is easy to see that, a Rolewicz-type operator $\lambda T_{f}$ is hypercyclic if and only if $|\lambda|>1$.

The aim of this paper is to study joint dynamics of tuples of Rolewicz-type operators on $c_{0}(\mathbb{N})$ and $\ell^{p}(\mathbb{N}), 1 \leq p<\infty$. In particular, we will characterize disjoint hypercyclic and simultaneous hypercyclic Rolewicz-type operators on these spaces.

Disjointness in hypercyclicity is introduced independently by Bernal [2] and by Bès and Peris [5] in 2007. For $N \geq 2$, operators $T_{1}, \ldots, T_{N} \in \mathcal{B}(X)$ are called disjoint hypercyclic or d-hypercyclic if the direct sum operator $T_{1} \oplus \cdots \oplus T_{N}$ has a hypercyclic vector of the form $(x, \ldots, x) \in X^{N}$. Such a vector $x \in X$ is called a d-hypercyclic vector for $T_{1}, \ldots, T_{N}$. If the set of d-hypercyclic vectors of $T_{1}, \ldots, T_{N}$ is dense, then $T_{1}, \ldots, T_{N}$ are called to be densely d-hypercyclic.

The similar and weaker notion of simultaneous hypercyclicity is introduced and studied by Bernal and Jung [3] in 2018. Among many examples, they gave a characterization for simultaneous hypercyclicity of different powers of weighted shifts.

Definition 1.1. [3, Definition 2.1] For $N \geq 2$, the operators $T_{1}, \ldots, T_{N} \in \mathcal{B}(X)$ are called simultaneously hypercyclic (or s-hypercyclic) if there exists $x \in X$ such that

$$
\overline{\left\{\left(T_{1}^{n} x, \ldots, T_{N}^{n} x\right): n \in \mathbb{N}\right\}} \supset \Delta\left(X^{N}\right),
$$

where $\Delta\left(X^{N}\right):=\{(x, x, \ldots, x): x \in X\}$ denotes the diagonal of $X^{N}$. Such a vector $x$ is said to be a s-hypercyclic vector of $T_{1}, \ldots, T_{N}$. If the set of s-hypercyclic vectors of $T_{1}, \ldots, T_{N}$ is dense in $X$, then $T_{1}, \ldots, T_{N} \in \mathcal{B}(X)$ are called as densely s-hypercyclic.

In Section 2, we will charcterize disjoint hypercyclic and disjoint supercyclic (see the definition in the beginning of Section 2) Rolewicz-type operators on $c_{0}$ and $\ell^{p}(\mathbb{N}), 1 \leq p<$ $\infty$. In Section 3, we will give a characterization for simultaneous hypercyclic Rolewicz-type operators.

For more on disjoint hypercyclic and disjoint supercyclic weighted shifts, one can see $[4,5,9,10]$. For more on hypercyclic operators and chaotic linear dynamics, one can see the books [1] and [8].

In the rest of the Introduction, we introduce the notation and results that we will use throughout the paper.

For any positive integer $n$, we define $[n]:=\{1,2, \ldots, n\}, f(A):=\{f(n): n \in A\}$ for $A \subset \mathbb{N}, f^{n}:=f \circ \ldots \circ f$ which is $f$ composed with itself $n$ many times, and $f^{-n}$ as the inverse of $f^{n}$ defined on its image $f^{n}(\mathbb{N})$.

One way to prove the d-hypercyclicity of a tuple of operators is to show that they are d-topologically transitive. $T_{1}, \ldots, T_{N} \in \mathcal{B}(X)$ are called as d-topologically transitive if for any non-empty open sets $U, V_{1}, \ldots, V_{N} \subset X$, there exists a positive integer $n$ such that $U \cap T_{1}^{-n}\left(V_{1}\right) \cap \ldots \cap T_{N}^{-n}\left(V_{N}\right) \neq \emptyset$. Bès and Peris [5] proved that operators $T_{1}, \ldots, T_{N}$ are densely d-hypercyclic if and only if they are d-topologically transitive. In [13], contrary to the single operator case, Sanders and Shkarin showed the existence of d-hypercyclic operators which are not densely d-hypercyclic and, therefore, fail to be d-topologically transitive.

Next, we recall another necessary condition for d-hypercyclicity, a natural extension of the Hypercyclicity Criterion which has played a significant role in linear dynamics.

Definition 1.2. Let $\left(n_{k}\right)$ be a strictly increasing sequence of positive integers. We say that $T_{1}, \ldots, T_{N} \in \mathcal{B}(X)$ satisfy the Disjoint Hypercyclicity Criterion with respect to $\left(n_{k}\right)$ 
provided there exist dense subsets $X_{0}, X_{1}, \ldots, X_{N}$ of $X$ and mappings $S_{m, k}: X_{m} \rightarrow X$ with $1 \leq m \leq N, k \in \mathbb{N}$ satisfying

$$
\begin{aligned}
T_{m}^{n_{k}} \underset{k \rightarrow \infty}{\longrightarrow} 0 & \text { pointwise on } X_{0}, \\
S_{m, k} \underset{k \rightarrow \infty}{\longrightarrow} 0 & \text { pointwise on } X_{m} \text {, and } \\
\left(T_{m}^{n_{k}} S_{i, k}-\delta_{i, m} I d_{X_{m}}\right) \underset{k \rightarrow \infty}{\longrightarrow} 0 & \text { pointwise on } X_{m}(1 \leq i \leq N) .
\end{aligned}
$$

In general, we say that $T_{1}, \ldots, T_{N}$ satisfy the d-Hypercyclicity Criterion if there exists some sequence $\left(n_{k}\right)$ for which (1.1) is satisfied.

Proposition 1.3. [5, Theorem 2.7] $T_{1}, T_{2}, \ldots, T_{N} \in \mathcal{B}(X)$ satisfy the Disjoint Hypercyclicity Criterion with respect to a sequence $\left(n_{k}\right)$ if and only if for each $r \in \mathbb{N}$, the direct sum operators $\bigoplus_{j=1}^{r} T_{1}, \ldots, \bigoplus_{j=1}^{r} T_{N}$ are d-topologically mixing on $X^{N}$. In particular, this implies that $T_{1}, \ldots, T_{N}$ are densely d-hypercyclic.

We now state an analogous criterion for simultaneous hypercyclicity from [3]. Recall that the convex hull $\operatorname{conv}(A)$ of a subset $A$ of a vector space $X$ is the smallest convex subset of $X$ containing $A$.

Definition 1.4. [3, Definition 3.6] Let $\left(n_{k}\right) \subset \mathbb{N}$ be a strictly increasing sequence and $T_{j} \in \mathcal{B}(X)(j=1, \ldots, N)$. We say that $T_{1}, \ldots, T_{N}$ satisfy the Simultaneous Hypercyclicity Criterion with respect to $\left(n_{k}\right)$ if there are subsets $X_{0} \subset X$ and $W_{0} \subset X^{N}$ such that $X_{0}$ is dense in $X$ and

$$
\overline{W_{0}} \supset \Delta\left(X^{N}\right)
$$

as well as mappings $R_{k}: W_{0} \rightarrow X(k \in \mathbb{N})$ such that

(i) $T_{j}^{n_{k}} \rightarrow 0$ pointwise on $X_{0}$ as $k \rightarrow \infty(j=1, \ldots, N)$,

(ii) $R_{k} \rightarrow 0$ pointwise on $W_{0}$ as $k \rightarrow \infty$, and

(iii) for every $w=\left(w_{1}, \ldots, w_{N}\right) \in W_{0}$ and every $j \in\{1, \ldots, N\}$ there is $y_{j} \in$ $\operatorname{conv}\left(\left\{w_{1} \ldots, w_{N}\right\}\right)$ such that $T_{j}^{n_{k}} R_{k} w \rightarrow y_{j}$ as $k \rightarrow \infty$.

Proposition 1.5. [3, Theorem 3.7] Let $T_{1}, \ldots, T_{N} \in \mathcal{B}(X)$. If $T_{1}, \ldots, T_{N}$ satisfy the Simultaneous Hypercyclicity Criterion with respect to some $\left(n_{k}\right) \subset \mathbb{N}$, then $T_{1}, \ldots, T_{N}$ are densely s-hypercyclic.

\section{Disjoint hypercyclic Rolewicz-type operators}

In this section, we give a full characterization for d-hypercyclicity and d-supercyclicity of Rolewicz-type operators and show that these two notions are equivalent among a subfamily of these operators. Recall that $T \in \mathcal{B}(X)$ is called supercyclic if there exists a vector $x \in X$ such that the set $\left\{\lambda T^{n} x: \lambda \in \mathbb{K}\right.$ and $\left.n \in \mathbb{N}\right\}$ is dense in $X$. Such a vector $x$ is said to be a supercyclic vector for $T$.

For $N \geq 2$, operators $T_{1}, \ldots, T_{N} \in \mathcal{B}(X)$ are called disjoint supercyclic or d-supercyclic if the direct sum operator $T_{1} \oplus \cdots \oplus T_{N}$ has a supercyclic vector of the form $(x, \ldots, x) \in X^{N}$. Such a vector $x \in X$ is called a $d$-supercyclic vector for $T_{1}, \ldots, T_{N}$. If the set of dsupercyclic vectors of $T_{1}, \ldots, T_{N}$ is dense, then $T_{1}, \ldots, T_{N}$ are called to be densely $d$ supercyclic.

Definition 2.1. Let $\left(n_{k}\right)$ be a strictly increasing sequence of positive integers. The operators $T_{1}, \ldots, T_{N} \in \mathcal{B}(X)$ are said to satisfy the Disjoint Supercyclicity Criterion with respect to the sequence $\left(n_{k}\right)$ if there exist dense subsets $X_{0}, X_{1}, \ldots, X_{N}$ of $X$, a sequence $\left(\mu_{k}\right)$ in $\mathbb{K}^{\mathbb{N}}$, and mappings $S_{m, k}: X_{m} \rightarrow X$ with $1 \leq m \leq N, k \in \mathbb{N}$ so that for each 
$1 \leq m \leq N$ we have

$$
\begin{aligned}
\mu_{k} T_{m}^{n_{k}} \underset{k \rightarrow \infty}{\longrightarrow} 0 & \text { pointwise on } X_{0}, \\
\frac{1}{\mu_{k}} S_{m, k} \underset{k \rightarrow \infty}{\longrightarrow} 0 & \text { pointwise on } X_{m} \text {, and } \\
\left(T_{m}^{n_{k}} S_{i, k}-\delta_{i, m} I d_{X_{m}}\right) \underset{k \rightarrow \infty}{\longrightarrow} 0 & \text { pointwise on } X_{m}(1 \leq i \leq N) .
\end{aligned}
$$

Proposition 2.2. [10, Proposition 1.11] Let $T_{1}, T_{2}, \ldots, T_{N}$ be operators on $X$ that satisfy the Disjoint Supercyclicity Criterion with respect to a sequence $\left(n_{k}\right)$. Then, $T_{1}, \ldots, T_{N}$ are densely d-supercyclic.

Now, we can give our first characterization.

Theorem 2.3. Let $\lambda_{1} T_{f_{1}}, \ldots, \lambda_{N} T_{f_{N}}$ be Rolewicz-type operators on $c_{0}$ or $\ell^{p}(\mathbb{N}), 1 \leq p<$ $\infty$, with $1<\left|\lambda_{1}\right| \leq \cdots \leq\left|\lambda_{N}\right|$. Then, the following are equivalent:

(i) $\lambda_{1} T_{f_{1}}, \ldots, \lambda_{N} T_{f_{N}}$ are $d$-supercyclic.

(ii) $\lambda_{1} T_{f_{1}}, \ldots, \lambda_{N} T_{f_{N}}$ are d-hypercyclic.

(iii) $\lambda_{1} T_{f_{1}}, \ldots, \lambda_{N} T_{f_{N}}$ are densely d-hypercyclic.

(iv) $\lambda_{1} T_{f_{1}}, \ldots, \lambda_{N} T_{f_{N}}$ satisfy the Disjoint Hypercyclicity Criterion.

(v) For any $k \in \mathbb{N}$ there exists arbitrarily large $n \in \mathbb{N}$ such that

(a) if $1 \leq t<s \leq N$ with $\left|\lambda_{t}\right|<\left|\lambda_{s}\right|$, then $f_{t}^{n}([k]) \cap f_{s}^{n}(\mathbb{N})=\emptyset$, and

(b) if $1 \leq t<s \leq N$ with $\left|\lambda_{t}\right|=\left|\lambda_{s}\right|$, then $f_{t}^{n}([k]) \cap f_{s}^{n}(\mathbb{N})=f_{s}^{n}([k]) \cap f_{t}^{n}(\mathbb{N})=\emptyset$.

Proof. The implications (iii) $\Longrightarrow$ (ii) $\Longrightarrow$ (i) are obvious and (iv) $\Longrightarrow$ (iii) follows from Proposition 1.3.

(i) $\Longrightarrow(\mathrm{v})$ :

Let $k \in \mathbb{N}$ and $x=\left(x_{1}, x_{2}, \ldots\right)$ be a d-supercyclic vector for $\lambda_{1} T_{f_{1}}, \ldots, \lambda_{N} T_{f_{N}}$. Choose $0 \neq \alpha \in \mathbb{K}$ and a large enough $n \in \mathbb{N}$ such that for all $i \in[N]$ we have

$$
\left\|\alpha \lambda_{i}^{n} T_{f_{i}}^{n} x-i\left(e_{1}+\cdots+e_{k}\right)\right\|<\delta
$$

where $1 / 2>\delta>0$ satisfies

$$
\frac{t}{s}-\frac{1}{3 N}<\frac{t-\delta}{s+\delta}<\frac{t+\delta}{s-\delta}<\frac{t}{s}+\frac{1}{3 N}
$$

and

$$
\frac{\delta}{s-\delta}<\frac{1}{3 N}
$$

for any $1 \leq t, s \leq N$, and $n$ satisfies

$$
\left|\frac{\lambda_{s}}{\lambda_{t}}\right|^{n} \frac{s-\delta}{s+\delta}>2 N
$$

for any $1 \leq t<s \leq N$ with $\left|\lambda_{t}\right|<\left|\lambda_{s}\right|$.

Observe that, by (2.2), we have that for all $t \in[N]$ and $j \in[k]$

$$
t-\delta<\left|\alpha \lambda_{t}^{n} x_{f_{t}^{n}(j)}\right|<t+\delta
$$

and for $j>k$

$$
\left|\alpha \lambda_{t}^{n} x_{f_{t}^{n}(j)}\right|<\delta .
$$

Now assume $\left|\lambda_{t}\right|<\left|\lambda_{s}\right|$ for some $1 \leq t<s \leq N$ and, by way of contradiction, suppose that $f_{t}^{n}\left(j_{0}\right) \in f_{s}^{n}(\mathbb{N})$ for some $j_{0} \in[k]$. We have two cases:

Case 1. Assume $\ell:=f_{s}^{-n}\left(f_{t}^{n}\left(j_{0}\right)\right) \in[k]$. Then, by (2.3), (2.5), and (2.6), 


$$
\begin{aligned}
N+\frac{1}{3 N}>\frac{s+\delta}{t-\delta}>\frac{\left|\alpha \lambda_{s}^{n} x_{f_{s}^{n}\left(j_{0}\right)}\right|}{\left|\alpha \lambda_{t}^{n} x_{f_{t}^{n}\left(j_{0}\right)}\right|} & =\left|\frac{\lambda_{s}}{\lambda_{t}}\right|^{n} \frac{\left|\alpha \lambda_{s}^{n} x_{f_{s}^{n}\left(j_{0}\right)}\right|}{\left|\alpha \lambda_{s}^{n} x_{f_{s}^{n}\left(f_{s}^{-n}\left(f_{t}^{n}\left(j_{0}\right)\right)\right)}\right|} \\
& >\left|\frac{\lambda_{s}}{\lambda_{t}}\right|^{n} \frac{\left|\alpha \lambda_{s}^{n} x_{f_{s}^{n}\left(j_{0}\right)}\right|}{\left|\alpha \lambda_{s}^{n} x_{f_{s}^{n}(\ell)}\right|} \\
& >\left|\frac{\lambda_{s}}{\lambda_{t}}\right|^{n} \frac{s-\delta}{s+\delta}>2 N
\end{aligned}
$$

which is a contradition.

Case 2. Assume $\ell:=f_{s}^{-n}\left(f_{t}^{n}\left(j_{0}\right)\right)>k$. Then, by (2.3), (2.4), (2.6), and (2.7),

$$
\begin{aligned}
\frac{1}{N}-\frac{1}{3 N}<\frac{t-\delta}{s+\delta}<\frac{\left|\alpha \lambda_{t}^{n} x_{f_{t}^{n}\left(j_{0}\right)}\right|}{\left|\alpha \lambda_{s}^{n} x_{f_{s}^{n}\left(j_{0}\right)}\right|} & =\left|\frac{\lambda_{t}}{\lambda_{s}}\right|^{n} \frac{\left|\alpha \lambda_{s}^{n} x_{f_{s}^{n}\left(f_{s}^{-n}\left(f_{t}^{n}\left(j_{0}\right)\right)\right)}\right|}{\left|\alpha \lambda_{s}^{n} x_{f_{s}^{n}\left(j_{0}\right)}\right|} \\
& <\frac{\left|\alpha \lambda_{s}^{n} x_{f_{s}^{n}(\ell)}\right|}{\left|\alpha \lambda_{s}^{n} x_{f_{s}^{n}\left(j_{0}\right)}\right|} \\
& <\frac{\delta}{s-\delta}<\frac{1}{3 N},
\end{aligned}
$$

which is again a contradition. Therefore, we can conclude that $f_{t}^{n}([k]) \cap f_{s}^{n}(\mathbb{N})=\emptyset$.

Now let $\left|\lambda_{t}\right|=\left|\lambda_{s}\right|$ for some $1 \leq t<s \leq N$ and, by way of contradiction, assume that $f_{t}^{n}\left(j_{0}\right) \in f_{s}^{n}(\mathbb{N})$ or $f_{s}^{n}\left(j_{0}\right) \in f_{t}^{n}(\mathbb{N})$ for some $j_{0} \in[k]$. Both of these assumptions will lead us to a similar contradiction so it is enough to consider the first one. Again, we have two cases. Assuming that $\ell:=f_{s}^{-n}\left(f_{t}^{n}\left(j_{0}\right)\right) \in[k]$, by similar calculations above and by $(2.3)$, (2.5), and (2.6), we have

$$
\frac{N-1}{N}+\frac{1}{3 N}>\frac{\left|\alpha \lambda_{t}^{n} x_{f_{t}^{n}\left(j_{0}\right)}\right|}{\left|\alpha \lambda_{s}^{n} x_{f_{s}^{n}\left(j_{0}\right)}\right|}>1-\frac{1}{3 N},
$$

which gives us a contradition. On the other hand, assuming $\ell:=f_{s}^{-n}\left(f_{t}^{n}\left(j_{0}\right)\right)>k$, by (2.3), (2.4), (2.6), and (2.7), we have

$$
\frac{1}{N}-\frac{1}{3 N}<\frac{\left|\alpha \lambda_{t}^{n} x_{f_{t}^{n}\left(j_{0}\right)}\right|}{\left|\alpha \lambda_{s}^{n} x_{f_{s}^{n}\left(j_{0}\right)}\right|}<\frac{1}{3 N}
$$

which again gives a contradition and finishes the proof of the implication.

(v) $\Longrightarrow($ iv):

Let $X=\left\{x^{(k)}: k \in \mathbb{N}\right\}$ be a countable dense set in $\ell^{p}(\mathbb{N})$ such that $x^{(k)} \in \operatorname{span}\left\{e_{1}, \ldots, e_{k}\right\}$. Then for $1 \leq j \leq N$ and for all $x \in X$, we have $\lambda_{j}^{n} T_{f_{j}}^{n} x \rightarrow 0$ as $n \rightarrow \infty$. Now we define $S_{j}:=\frac{1}{\lambda_{j}} F_{f_{j}}$ where $F_{f_{j}}: \ell^{p}(\mathbb{N}) \rightarrow \ell^{p}(\mathbb{N})$ is given as

$$
F_{f_{j}}\left(x_{1}, x_{2}, \ldots\right):=\left(0, \ldots, 0, x_{1}, 0, \ldots, 0, x_{2}, 0, \ldots\right),
$$

where $x_{k}$ is in the $f(k)^{t h}$ position, for $k \in \mathbb{N}$. Since $\left|\lambda_{j}\right|>1, S_{j} x \rightarrow 0$ as $n \rightarrow \infty$ and $\lambda_{j} T_{f_{j}} S_{j} x=x$ for $1 \leq j \leq N$ and for all $x \in X$.

Observe that for $1 \leq t, s \leq N$

$$
\lambda_{t}^{n} T_{f_{t}}^{n} S_{s}^{n} e_{k}=\left(\frac{\lambda_{t}}{\lambda_{s}}\right)^{n} T_{f_{t}}^{n} F_{f_{s}}^{n} e_{k}=\left\{\begin{array}{cl}
\left(\frac{\lambda_{t}}{\lambda_{s}}\right)^{n} e_{f_{t}^{[-n]}\left(f_{s}^{[n]}(k)\right),} & \text { if } f_{s}^{[n]}(k) \in \operatorname{Im}\left(f_{t}^{[n]}\right), \\
0, & \text { otherwise. }
\end{array}\right.
$$

Now, for each $k \in \mathbb{N}$ we choose a big enough $n_{k} \in \mathbb{N}$ such that for $1 \leq t<s \leq N$ with $\left|\lambda_{t}\right|=\left|\lambda_{s}\right|$ we have $\lambda_{t}^{n_{k}} T_{f_{t}}^{n_{k}} S_{s}^{n_{k}} x^{(k)}=\lambda_{s}^{n_{k}} T_{f_{s}}^{n_{k}} S_{t}^{n_{k}} x^{(k)}=0$ and for $1 \leq t<s \leq N$ with $\left|\lambda_{t}\right|<\left|\lambda_{s}\right|$ we have $\lambda_{s}^{n_{k}} T_{f_{s}}^{n_{k}} S_{t}^{n_{k}} x^{(k)}=0$ and

$$
\left\|\lambda_{t}^{n_{k}} T_{f_{t}}^{n_{k}} S_{s}^{n_{k}} x^{(k)}\right\| \leq\left|\frac{\lambda_{t}}{\lambda_{s}}\right|^{n_{k}}\left\|x^{(k)}\right\|<\frac{1}{k} .
$$


Therefore, it is easy to see that $\lambda_{1} T_{f_{1}}, \ldots, \lambda_{N} T_{f_{N}}$ satisfy the Disjoint Hypercyclicity Criterion with respect to the sequence $\left(n_{k}\right)_{k=1}^{\infty}$ where $X_{j}=X$ and $S_{j, n}=S_{j}^{n}$ for $1 \leq j \leq$ $N$.

Recall that $T_{1}, T_{2}$ are said to be $d$-weakly mixing if the direct sums $T_{1} \oplus T_{1}, T_{2} \oplus T_{2}$ are d-topologically transitive on $X \times X$.

Example 2.4. Let $T_{1}=\lambda B=\lambda T_{f_{1}}$ and $T_{2}=\mu B^{2}=\mu T_{f_{2}}$, where $f_{1}(n)=n+1$ and $f_{2}(n)=n+2$ for $n \in \mathbb{N}$. The following assertions are equivalent:

(1) the operators $T_{1}$ and $T_{2}$ are d-hypercyclic;

(2) the operators $T_{1}$ and $T_{2}$ are d-weakly mixing;

(3) $1<|\lambda|<|\mu|$.

It is easy to see this using Theorem 2.3 and Proposition 1.3.

Example 2.5. Let $f_{1}, f_{2}: \mathbb{N} \rightarrow \mathbb{N}$ be defined as $f_{1}(n)=2 n$ and $f_{2}(n)=2 n+1$ for $n \in \mathbb{N}$. Then, since $f_{1}^{n}(\mathbb{N}) \cap f_{2}^{n}(\mathbb{N})=\emptyset$ for all $n \in \mathbb{N}, \lambda T_{f_{1}}, \mu T_{f_{2}}$ are d-hypercyclic if and only if $|\lambda|,|\mu|>1$.

Of course, the condition $\left|\lambda_{1}\right|, \ldots,\left|\lambda_{N}\right|>1$ is not necessary for d-supercyclicity. For non-zero scalars, we can give the following characterization:

Theorem 2.6. Let $\lambda_{1} T_{f_{1}}, \ldots, \lambda_{N} T_{f_{N}}$ be Rolewicz-type operators on $c_{0}$ or $\ell^{p}(\mathbb{N}), 1 \leq p<$ $\infty$, with $0<\left|\lambda_{1}\right| \leq \cdots \leq\left|\lambda_{N}\right|$. Then, the following are equivalent:

(i) $\lambda_{1} T_{f_{1}}, \ldots, \lambda_{N} T_{f_{N}}$ are $d$-supercyclic.

(ii) $\lambda_{1} T_{f_{1}}, \ldots, \lambda_{N} T_{f_{N}}$ are densely d-supercyclic.

(iii) $\lambda_{1} T_{f_{1}}, \ldots, \lambda_{N} T_{f_{N}}$ satisfy the d-Supercyclicity Criterion.

(iv) For any $k \in \mathbb{N}$ there exists arbitrarily large $n \in \mathbb{N}$ such that

(a) if $1 \leq t<s \leq N$ with $\left|\lambda_{t}\right|<\left|\lambda_{s}\right|$, then $f_{t}^{n}([k]) \cap f_{s}^{n}(\mathbb{N})=\emptyset$, and

(b) if $1 \leq t<s \leq N$ with $\left|\lambda_{t}\right|=\left|\lambda_{s}\right|$, then $f_{t}^{n}([k]) \cap f_{s}^{n}(\mathbb{N})=f_{s}^{n}([k]) \cap f_{t}^{n}(\mathbb{N})=\emptyset$.

Proof. (i) $\Longrightarrow$ (iv):

The proof of this implication is just like the proof of the implication (i) $\Longrightarrow(v)$ in Theorem 2.3 since the condition $\left|\lambda_{1}\right|, \ldots,\left|\lambda_{N}\right|>1$ is not used in the proof.

(iv) $\Longrightarrow$ (iii):

Let $\mu=\max \left\{\frac{1}{\lambda_{1}}, \ldots, \frac{1}{\lambda_{N}}\right\}$ and $\left(\mu_{k}\right)_{k \in \mathbb{N}}=\left((1+\mu)^{k}\right)_{k \in \mathbb{N}}$. Let $X=\left\{x^{(k)}: k \in \mathbb{N}\right\}$ be a countable dense set in $\ell^{p}(\mathbb{N})$ such that $x^{(k)} \in \operatorname{span}\left\{e_{1}, \ldots, e_{k}\right\}$. Then, for $1 \leq j \leq N$ and for all $x \in X$, we have $\mu_{n} \lambda_{j}^{n} T_{f_{j}}^{n} x \rightarrow 0$ as $n \rightarrow \infty$.

Now we define $S_{j}:=\frac{1}{\lambda_{j}} F_{f_{j}}$, where $F_{f_{j}}: \ell^{p}(\mathbb{N}) \rightarrow \ell^{p}(\mathbb{N})$ is defined as in (2.8). Thus, $\frac{1}{\mu_{n}} S_{j}^{n} \rightarrow 0$ as $n \rightarrow \infty$ and $\lambda_{j} T_{f_{j}} S_{j} x=x$ for $1 \leq j \leq N$ and for all $x \in X$.

Now, by similar calculations as in the proof of the implication (v) $\Longrightarrow$ (iv) in Theorem 2.3 , we can see that for each $k \in \mathbb{N}$ we choose a big enough $n_{k} \in \mathbb{N}$ such that for $1 \leq$ $t<s \leq N$ with $\left|\lambda_{t}\right|=\left|\lambda_{s}\right|$, we have $\lambda_{t}^{n_{k}} T_{f_{t}}^{n_{k}} S_{s}^{n_{k}} x^{(k)}=\lambda_{s}^{n_{k}} T_{f_{s}}^{n_{k}} S_{t}^{n_{k}} x^{(k)}=0$. Also, for $1 \leq t<s \leq N$ with $\left|\lambda_{t}\right|<\left|\lambda_{s}\right|$, we have $\lambda_{s}^{n_{k}} T_{f_{s}}^{n_{k}} S_{t}^{n_{k}} x^{(k)}=0$ and $\left\|\lambda_{t}^{n_{k}} T_{f_{t}}^{n_{k}} S_{s}^{n_{k}} x^{(k)}\right\|<\frac{1}{k}$.

Therefore, we see that $\lambda_{1} T_{f_{1}}, \ldots, \lambda_{N} T_{f_{N}}$ satisfy the Disjoint Supercyclicity Criterion with respect to the sequences $\left(n_{k}\right)_{k=1}^{\infty}$ in $\mathbb{N}$ and $\left(\mu_{k}\right)_{k=1}^{\infty}$ in $\mathbb{K}$, where $X_{j}=X$ and $S_{j, n}=S_{j}^{n}$ for $1 \leq j \leq N$.

(iii) $\Longrightarrow$ (ii) follows from Proposition 2.2 and the implication (ii) $\Longrightarrow$ (i) is obvious.

\section{Simultaneous hypercyclic Rolewicz-type operators}

In this section, we characterize simultaneous hypercyclic Rolewicz-type operators.

Theorem 3.1. Let $\lambda_{1} T_{f_{1}}, \ldots, \lambda_{N} T_{f_{N}}$ be Rolewicz-type operators on $c_{0}$ or $\ell^{p}(\mathbb{N}), 1 \leq p<$ $\infty$, with $1<\left|\lambda_{1}\right| \leq \cdots \leq\left|\lambda_{N}\right|$. Then, the following are equivalent: 
(i) $\lambda_{1} T_{f_{1}}, \ldots, \lambda_{N} T_{f_{N}}$ are s-hypercyclic.

(ii) $\lambda_{1} T_{f_{1}}, \ldots, \lambda_{N} T_{f_{N}}$ are densely s-hypercyclic.

(iii) $\lambda_{1} T_{f_{1}}, \ldots, \lambda_{N} T_{f_{N}}$ satisfy the Simultaneous Hypercyclicity Criterion.

(iv) For every $\varepsilon>0$ and for every $k \in \mathbb{N}$ there exists arbitrarily large $n_{k} \in \mathbb{N}$ such that

(a) if $1 \leq t<s \leq N$ with $\left|\lambda_{t}\right|<\left|\lambda_{s}\right|$, then $f_{t}^{n_{k}}([k]) \cap f_{s}^{n_{k}}(\mathbb{N})=\emptyset$.

(b) if $1 \leq t<s \leq N$ with $\left|\lambda_{t}\right|=\left|\lambda_{s}\right|$, then

$$
f_{t}^{n_{k}}([k]) \cap f_{s}^{n_{k}}(\mathbb{N} \backslash[k])=f_{s}^{n_{k}}([k]) \cap f_{t}^{n_{k}}(\mathbb{N} \backslash[k])=\emptyset
$$

and one of the following is satisfied

(b1) $f_{t}^{n_{k}}([k]) \cap f_{s}^{n_{k}}([k])=\emptyset$,

(b2) for every $\ell \in f_{t}^{n_{k}}([k]) \cap f_{s}^{n_{k}}([k])$,

$$
f_{t}^{-n_{k}}(\ell)=f_{s}^{-n_{k}}(\ell)
$$

and

$$
\left|\left(\frac{\lambda_{t}}{\lambda_{s}}\right)^{n_{k}}-1\right|<\varepsilon
$$

Proof. (i) $\Longrightarrow$ (iv):

Let $\varepsilon>0, k \in \mathbb{N}$ and let $x=\left(x_{1}, x_{2}, \ldots\right)$ be a s-hypercyclic vector for $\lambda_{1} T_{f_{1}}, \ldots, \lambda_{N} T_{f_{N}}$. Choose a large enough $n_{k}$ such that for $i=1,2, \ldots, N$ we have

$$
\left\|\lambda_{i}^{n_{k}} T_{f_{i}}^{n_{k}} x-\sum_{j=1}^{k} j e_{j}\right\|<\delta,
$$

where $0<\delta<\frac{1}{k+2}$ and for all $t, s \in\{1,2, \ldots, N\}, n_{k}$ satisfies

$$
\left(\frac{k+\delta}{1-\delta}\right)^{2}<\left|\frac{\lambda_{s}}{\lambda_{t}}\right|^{n_{k}}
$$

if $\left|\lambda_{t}\right|<\left|\lambda_{s}\right|$.

Observe that, by (3.1), we have that for $i=1,2, \ldots, N$ and $j \in[k]$

$$
j-\delta<\left|\lambda_{i}^{n_{k}} x_{f_{i}^{n_{k}(j)}}\right|<j+\delta
$$

and for $j>k$

$$
\left|\lambda_{i}^{n_{k}} x_{f_{i}^{n_{k}(j)}}\right|<\delta
$$

Now assume $\left|\lambda_{t}\right|<\left|\lambda_{s}\right|$ and, by way of contradiction, suppose that $f_{t}^{n_{k}}\left(j_{0}\right) \in f_{s}^{n_{k}}(\mathbb{N})$ for some $j_{0} \in[k]$. We have two cases:

Case 1. Assume $\ell:=f_{s}^{-n_{k}}\left(f_{t}^{n_{k}}\left(j_{0}\right)\right) \in[k]$. Then, by (3.2) and (3.3),

$$
\begin{aligned}
\frac{k+\delta}{1-\delta} \geq \frac{j_{0}+\delta}{j_{0}-\delta}>\frac{\left|\lambda_{s}^{n_{k}} x_{f_{s}^{n_{k}}\left(j_{0}\right)}\right|}{\left|\lambda_{t}^{n_{k}} x_{f_{t}^{n_{k}\left(j_{0}\right)}}\right|} & =\left|\frac{\lambda_{s}}{\lambda_{t}}\right|^{n_{k}}\left|\frac{\lambda_{s}^{n_{k}} x_{f_{s}^{n_{k}}\left(j_{0}\right)}}{\lambda_{s}^{n_{k}} x_{f_{s} n_{k}(\ell)}}\right| \\
& >\left|\frac{\lambda_{s}}{\lambda_{t}}\right|^{n_{k}} \frac{j_{0}-\delta}{\ell+\delta} \\
& >\left(\frac{k+\delta}{1-\delta}\right)^{2} \frac{1-\delta}{k+\delta} \\
& =\frac{k+\delta}{1-\delta}
\end{aligned}
$$

which is a contradition.

Case 2. Assume $\ell:=f_{s}^{-n_{k}}\left(f_{t}^{n_{k}}\left(j_{0}\right)\right)>k$. Then, by (3.3) and (3.4), 


$$
\begin{aligned}
\frac{1-\delta}{k+\delta} \leq \frac{j_{0}-\delta}{j_{0}+\delta}<\frac{\left|\lambda_{t}^{n_{k}} x_{f_{t}^{n_{k}}\left(j_{0}\right)}\right|}{\left|\lambda_{s}^{n_{k}} x_{f_{s}^{n_{k}}\left(j_{0}\right)}\right|} & =\left|\frac{\lambda_{t}}{\lambda_{s}}\right|^{n_{k}}\left|\frac{\lambda_{s}^{n_{k}} x_{f_{s}^{n_{k}}(\ell)}}{\lambda_{s}^{n_{k}} x_{f_{s} n_{k}\left(j_{0}\right)}}\right| \\
& <\frac{\delta}{j_{0}-\delta} \\
& \leq \frac{\delta}{1-\delta},
\end{aligned}
$$

which contradicts $\delta<\frac{1}{k+2}$.

Therefore, we can conclude that $f_{t}^{n_{k}}([k]) \cap f_{s}^{n_{k}}(\mathbb{N})=\emptyset$.

Now let $\left|\lambda_{t}\right|=\left|\lambda_{s}\right|$. Note that the reasoning in Case 2 works here too. Consequently, because of the symmetry, we have $f_{t}^{m_{k}}([k]) \cap f_{s}^{n_{k}}(\mathbb{N} \backslash[k])=f_{s}^{n_{k}}([k]) \cap f_{t}^{n_{k}}(\mathbb{N} \backslash[k])=\emptyset$.

Now, by way of contradiction, assume that $\ell \in f_{t}^{n_{k}}([k]) \cap f_{s}^{n_{k}}([k])$ and $j_{1}=f_{t}^{-n_{k}}(\ell)>$ $f_{s}^{-n_{k}}(\ell)=j_{2}$. Then, by (3.3),

$$
\frac{j_{1}-\delta}{j_{2}+\delta}<\frac{\left|\lambda_{t}^{n_{k}} x_{f_{t}^{n_{k}}\left(j_{1}\right)}\right|}{\left|\lambda_{s}^{n_{k}} x_{f_{s}^{n_{k}}\left(j_{2}\right)}\right|}=\left|\frac{\lambda_{t}}{\lambda_{s}}\right|^{n_{k}}\left|\frac{x_{\ell}}{x_{\ell}}\right|=1,
$$

which gives us a contradiction. Therefore, we conclude that $f_{t}^{-n_{k}}(\ell)=f_{s}^{-n_{k}}(\ell)$.

Now we have two cases:

If $f_{t}^{n_{k}}([k]) \cap f_{s}^{n_{k}}([k])=\emptyset$ for all $k \in \mathbb{N}$, then (iv)(b1) is satisfied.

Otherwise there exists a $k_{0}$ such that

$$
f_{t}^{n_{k}}\left(\left[k_{0}\right]\right) \cap f_{s}^{n_{k}}\left(\left[k_{0}\right]\right) \neq \emptyset \quad \text { for all } k \geq k_{0} .
$$

If this is not the case, then it is possible to choose a subsequence of $\left(n_{k}\right)$ for which (b1) is satisfied. By (3.5), there exist $j_{0} \in\left[k_{0}\right]$ and $\left(m_{k}\right) \subset\left(n_{k}\right)$ such that

$$
f_{t}^{m_{k}}\left(j_{0}\right)=f_{s}^{m_{k}}\left(j_{0}\right) \text { for all } k \geq k_{0} .
$$

By (3.1), it follows that as for all $k \geq k_{0}$

$$
j_{0}-\delta<\left|\lambda_{t}^{m_{k}} x_{f_{t}^{m_{k}\left(j_{0}\right)}}\right|<j_{0}+\delta
$$

and

Consequently,

$$
j_{0}-\delta<\left|\lambda_{s}^{m_{k}} x_{f_{s}^{m_{k}}\left(j_{0}\right)}\right|<j_{0}+\delta
$$

$$
\left|\left(\frac{\lambda_{t}}{\lambda_{s}}\right)^{m_{k}}-1\right|=\left|\frac{\lambda_{t}^{m_{k}} x_{f_{t}^{m_{k}}\left(j_{0}\right)}}{\lambda_{s}^{m_{k}} x_{f_{s}^{m_{k}}\left(j_{0}\right)}}-1\right|=\left|\frac{\lambda_{t}^{m_{k}} x_{f_{t}^{m_{k}}\left(j_{0}\right)}-j_{0}+j_{0}-\lambda_{s}^{m_{k}} x_{f_{s}^{m_{k}}\left(j_{0}\right)}}{\lambda_{s}^{m_{k}} x_{f_{s}^{m_{k}}\left(j_{0}\right)}}\right|<\frac{2 \delta}{j_{0}-\delta} .
$$

Then we rename $\left(m_{k}\right)$ as $\left(n_{k}\right)$ and (iv) is satisfied for $\left(n_{k}\right)$.

(iv) $\Longrightarrow$ (iii):

We will give the proof for two Rolewicz-type operators $\lambda_{1} T_{f_{1}}, \lambda_{2} T_{f_{2}}$ for brevity. The general case follows similarly.

If (iv)(a) is satisfied, then, from Theorem 2.3, it follows that $\lambda_{1} T_{f_{1}}, \lambda_{2} T_{f_{2}}$ satisfy the Disjoint Hypercyclicity Criterion and consequently, by [3, Remark 3.8.3], they satisfy the Simultaneous Hypercyclicity Criterion.

If conditions of (iv)(b) are satisfied for some strictly increasing sequence $\left(m_{k}\right)$, let $X:=$ $\ell^{p}(\mathbb{N})$ and $X_{0}$ be the set of finitely supported sequences in $X$. It follows that $\bar{X}_{0}=X$. Let $W_{0}:=\Delta\left(X_{0}^{2}\right) \subset X^{2}$. Note that $\overline{W_{0}}=\overline{\Delta\left(X_{0}^{2}\right)}=\Delta\left(X^{2}\right)$. Now we want to show that $\lambda_{1} T_{f_{1}}$, $\lambda_{2} T_{f_{2}}$ satisfy the conditions of the Simultaneous Hypercyclicity Criterion (Definition 1.4).

It is clear that $\left(\lambda_{i} T_{f_{i}}\right)^{m_{k}} \rightarrow 0$ pointwise on $X_{0}$ as $k \rightarrow \infty$ for $i=1,2$. Define, for any $k \in \mathbb{N}$, the mapping $R_{k}: W_{0} \rightarrow X$ as follows. Let $A_{k}:=\left\{j \in[k]: f_{1}^{m_{k}}(j)=f_{2}^{m_{k}}(j)\right\}$. 
If $x=\left(x_{1}, x_{2}, \ldots, x_{N}, 0,0 \ldots\right) \in X_{0}$ and $w=(x, x) \in W_{0}$, then

$$
R_{k} w:=\left\{\begin{array}{ll}
\sum_{j=1}^{N} \frac{x_{j}}{\lambda_{1}^{m_{k}}} e_{f_{1}^{m_{k}}(j)}+\sum_{\substack{j=1 \\
j \notin A_{k}}}^{N} \frac{x_{j}}{\lambda_{2}^{m_{k}}} e_{f_{2}^{m_{k}}(j)}, & k \geq N \\
0, & k<N
\end{array} .\right.
$$

Since $1<\left|\lambda_{1}\right|=\left|\lambda_{2}\right|, R_{k} \rightarrow 0$ pointwise on $W_{0}$ as $k \rightarrow \infty$.

Note that

$$
\left(\lambda_{1} T_{f_{1}}\right)^{m_{k}} R_{k} w=\sum_{j=1}^{N} \frac{x_{j}}{\lambda_{1}^{m_{k}}} \lambda_{1}^{m_{k}} e_{j}=\sum_{j=1}^{N} x_{j}=x
$$

so $\left(\lambda_{1} T_{f_{1}}\right)^{m_{k}} R_{k} w \rightarrow x$ as $k \rightarrow \infty$.

Note also that

$$
\left(\lambda_{2} T_{f_{2}}\right)^{m_{k}} R_{k} w=\sum_{\substack{j=1 \\ j \in A_{k}}}^{N} \frac{x_{j}}{\lambda_{1}^{m_{k}}} \lambda_{2}^{m_{k}} e_{j}+\sum_{\substack{j=1 \\ j \notin A_{k}}}^{N} \frac{x_{j}}{\lambda_{2}^{m_{k}}} \lambda_{2}^{m_{k}} e_{j}=\sum_{\substack{j=1 \\ j \in A_{k}}}^{N} x_{j}\left(\frac{\lambda_{2}}{\lambda_{1}}\right)^{m_{k}} e_{j}+\sum_{\substack{j=1 \\ j \notin A_{k}}}^{N} x_{j} e_{j} .
$$

If (b1) is satisfied then $A_{k}=\emptyset$ for all $k \in \mathbb{N}$ and $\left(\lambda_{2} T_{f_{2}}\right)^{m_{k}} R_{k} w=x$. Otherwise

$$
\left(\lambda_{2} T_{f_{2}}\right)^{m_{k}} R_{k} w \rightarrow x
$$

since $\left(\frac{\lambda_{1}}{\lambda_{2}}\right)^{m_{k}} \rightarrow 1$, as $k \rightarrow \infty$.

Therefore, $\lambda_{1} T_{f_{1}}, \lambda_{2} T_{f_{2}}$ satisfy the Simultaneous Hypercyclicity Criterion.

(iii) $\Longrightarrow$ (ii): This is true by Proposition 1.5.

(ii) $\Longrightarrow$ (i): This is obvious.

Example 3.2. Consider the Rolewicz-type operators $T_{1}=\lambda T_{f}$ and $T_{2}=\lambda e^{2 \pi i \alpha} T_{f}$, where $\lambda>1$ and $\alpha \in \mathbb{R}$. If $\alpha$ is rational, then there are infinitely many $n \in \mathbb{N}$ such that $e^{2 \pi i \alpha n}=1$. On the other hand, it is well known that the set $\left\{e^{2 \pi i \alpha n}: n \in \mathbb{N}\right\}$ is dense in the unit circle $\{z:|z|=1\} \subset \mathbb{C}$ if and only if $\alpha$ is irrational. Therefore, for any $\alpha$, there exists an increasing sequence $\left(n_{k}\right)$ of natural numbers such that $e^{2 \pi i \alpha n_{k}} \rightarrow 1$ as $k \rightarrow \infty$. Therefore, $T_{1}, T_{2}$ are s-hypercyclic. On the other hand, using Theorem 2.3, it is easy to see that these operators are not d-hypercyclic.

Corollary 3.3. [3, Proposition 5.3] For $1 \leq \ell \leq N$, let $\lambda_{\ell} \in \mathbb{K}$ and $r_{\ell} \in \mathbb{N}$ with $r_{1} \leq r_{2} \leq$ $\ldots \leq r_{N}$. Then, $\lambda_{1} B^{r_{1}}, \ldots, \lambda_{N} B^{r_{N}}$ is s-hypercyclic on $c_{0}$ or $\ell^{p}(\mathbb{N}), 1 \leq p<\infty$, if and only if

(1) $1<\left|\lambda_{j}\right|$ for all $j \in\{1, \ldots, N\}$,

(2) $\left|\lambda_{j}\right|<\left|\lambda_{j+1}\right|$ for all $j \in\{1, \ldots, N-1\} \backslash A$,

(3) $\left|\lambda_{j}\right|=\left|\lambda_{j+1}\right|$ for all $j \in A$,

where $A=\left\{j \in\{1, \ldots, N-1\}: r_{j}=r_{j+1}\right\}$.

Proof. Observe that $\lambda_{\ell} B^{r_{\ell}}=\lambda_{\ell} T_{f_{\ell}}$ where $f_{\ell}(n)=n+r_{\ell}$ for $1 \leq \ell \leq N$. First, assume that the conditions (1), (2), and (3) hold. For any $j \in\{1, \ldots, N-1\} \backslash A$ and $k \in \mathbb{N}$, let $n_{k}=k$. Then, for any $m \in[k]$ we have

$$
f_{j}^{n_{k}}(m)=m+k r_{j} \leq k+k r_{j}=k\left(r_{j}+1\right) \leq k r_{j+1} \notin\left\{n+k r_{j+1}: n \geq 1\right\}=f_{j+1}^{n_{k}}(\mathbb{N}) .
$$

Also, for $j \in A$, as argued in Example 3.2, there exists $\left(n_{k}\right)$ such that $\left(\lambda_{j+1} / \lambda_{j}\right)^{n_{k}} \rightarrow 1$. Thus, conditions of Theorem 3.1 are satisfied and $\lambda_{1} B^{r_{1}}, \ldots, \lambda_{N} B^{r_{N}}$ are s-hypercyclic.

Now assume that $\lambda_{1} B^{r_{1}}, \ldots, \lambda_{N} B^{r_{N}}$ are s-hypercyclic. In particular each $\lambda_{j} B^{r_{j}}$ is hypercyclic and $\left|\lambda_{j}\right|=\left|\lambda_{j}\right|\left\|B^{r_{j}}\right\|=\left\|\lambda_{j} B^{r_{j}}\right\|>1$. Using Theorem 3.1, it is easy to see the rest of the conditions are satisfied.

One can generalize Rolewicz-type operators further by multiplying the shifted vector with a weight sequence: 
Definition 3.4. Let $w$ be a weight sequence and let $f: \mathbb{N} \rightarrow \mathbb{N}$ be a strictly increasing map with $f(1) \neq 1$. The unilateral pseudo-shift $T_{f, w}$ on $c_{0}(\mathbb{N})$ or $\ell^{p}(\mathbb{N}), 1 \leq p<\infty$, is given by

$$
T_{f, w}\left(\sum_{j=1}^{\infty} x_{j} e_{j}\right)=\sum_{j=1}^{\infty} w_{f(j)} x_{f(j)} e_{j},
$$

where $\left\{e_{j}: j \geq 1\right\}$ is the canonical basis.

In 2000, Grosse-Erdmann [7] studied the chaotic dynamics of pseudo-shifts. Wang and Zhou [16], in 2018, characterized d-hypercyclicity of the tuples of pseudo-shifts of the form $T_{f, w_{1}}, \ldots, T_{f, w_{N}}$ which have the same inducing maps. In 2019, Wang and Liang [15] characterized d-supercyclicity of the tuples of pseudo-shifts of the same form. Wang, Chen and Zhou [14], also in 2019, characterized d-hypercyclicity and d-supercyclicity of tuples of pseudo-shifts of the form $T_{f_{1}, w_{1}}^{r_{1}}, \ldots, T_{f_{N}, w_{N}}^{r_{N}}$ where powers are pairwise distinct. Observe that none of these families cover the tuples of Rolewicz-type operators that we study in this paper.

We finish the paper with the following open question.

Question 3.5. Which pseudo-shifts (raised to the same power) are disjoint hypercyclic or simultaneous hypercyclic on $c_{0}(\mathbb{N})$ or $\ell^{p}(\mathbb{N}), 1 \leq p<\infty$ ?

Acknowledgment. The first author was partially supported by Istanbul Technical University Scientific Research Project [grant no. TAB-2017-40552]. The second author was partially supported by Mimar Sinan Fine Arts University Scientific Research Project [grant no. 2016-18].

\section{References}

[1] F. Bayart and E. Matheron, Dynamics of linear operators, Cambridge Tracts in Mathematics 179. Cambridge University Press, Cambridge, 2009.

[2] L. Bernal-González, Disjoint hypercyclic operators, Stud. Math. 182 (2), 113-130, 2007.

[3] L. Bernal-González and A. Jung, Simultaneous universality, J. Approx. Theory, 237, 43-65, 2018.

[4] J. Bès, Ö. Martin, and R. Sanders, Weighted shifts and disjoint hypercyclicity, J. Operator Theory, $\mathbf{7 2}$ (1), 15-40, 2014.

[5] J. Bès and A. Peris, Disjointness in hypercyclicity, J. Math. Anal. Appl. 336, 297-315, 2007.

[6] D. Bongiorno, U.B. Darji and L. Di Piazza, Rolewicz-type chaotic operators, J. Math Anal. Appl. 431 (1), 518-528, 2015.

[7] K.-G. Grosse-Erdmann, Hypercyclic and chaotic weighted shifts, Studia Math. 139 (1), 47-68, 2000.

[8] K.-G. Grosse-Erdmann and A. Peris, Linear chaos, Universitext: Tracts in mathematics. Springer, New York, 2011.

[9] Ö. Martin, Disjoint hypercyclic and supercyclic composition operators, $\mathrm{PhD}$ thesis, Bowling Green State University, 2010.

[10] Ö. Martin and R. Sanders, Disjoint supercyclic weighted shifts, Integr. Equ. Oper. Theory, 85, 191-220, 2016.

[11] S. Rolewicz, On orbits of elements, Studia Math. 32, 17-22, 1969.

[12] H. Salas, Hypercyclic weighted shifts, Trans. Amer. Math. Soc. 347 (3), 993-1004, 1995.

[13] R. Sanders and S. Shkarin, Existence of disjoint weakly mixing operators that fail to satisfy the Disjoint Hypercyclicity Criterion, J. Math. Anal. Appl. 417, 834-855, 2014 . 
[14] Y. Wang, C. Chen, and Z-H. Zhou, Disjoint hypercyclic weighted pseudoshift operators generated by different shifts, Banach J. Math. Anal. 13 (4), 815-836, 2019.

[15] Y. Wang and Y-X Liang, Disjoint supercyclic weighted pseudo-shifts on Banach sequence spaces, Acta Math. Sci. 39B (4), 1089-1102, 2019.

[16] Y. Wang and Z-H. Zhou, Disjoint hypercyclic weighted pseudo-shifts on Banach sequence spaces, Collect. Math. 69, 437-449, 2018. 